\title{
Co-Author Networks in Journal of the Korean Academy of Child and Adolescent Psychiatry
}

\author{
Soungwan $\mathrm{Kim}^{1}$, Bum-Sung $\mathrm{Choi}^{2}$, Bongseog Kim${ }^{1}$, and Kyoung-Min $\mathrm{Kim}^{3}$ \\ ${ }^{1}$ Department of Psychiatry, Inje University Sanggye Paik Hospital, Seoul, Korea \\ ${ }^{2}$ Department of Psychiatry, Pusan National University Yangsan Hospital, Yangsan, Korea \\ ${ }^{3}$ Division of Child and Adolescent Psychiatry, Department of Psychiatry, Seoul National University Hospital, Seoul, Korea
}

\section{학술지 소아청소년정신의학의 공저 네트워크 분석}

\author{
김성완 ${ }^{1}$, 최범성 ${ }^{2}$, 김봉석 ${ }^{1}$, 김경민 ${ }^{3}$ \\ 인제대학교 상계백병원 정신건강의학과, ${ }^{1}$ 양산부산대학교병원 정신건강의학과, ${ }^{2}$ 서울대학교병원 정신건강의학과 소아청소년정신분과 ${ }^{3}$
}

\begin{abstract}
Objectives: The purpose of this study is to analyze the co-author networks in the Journal of the Korean Academy of Child and Adolescent Psychiatry, a representative journal published by a branch of the domestic psychiatric academy, in order to present the current state of the co-authoring of and developments in child and adolescent psychiatry.

Methods: We visualized and estimated the basic characteristics of the co-author networks shown by 564 authors who wrote 251 papers published in the Journal of the Korean Academy of Child and Adolescent Psychiatry between 2005 and 2015, in order to assess their network characteristics, author centrality, and relevance to research performance.

Results: The co-author networks in the Journal of the Korean Academy of Child and Adolescent Psychiatry showed the characteristics of a small world and scale-free network. There was a correlation between the author centrality within the network and the research performance of the authors, but less correlation was shown between the centrality and mean paper citation counts.

Conclusion: The network structure in the Journal of the Korean Academy of Child and Adolescent Psychiatry showed similarity to the coauthoring of other branches. However, given that the mean paper citation counts were less correlated with the author centrality than those in other branches, it may be necessary to promote an increase in the mean paper citation counts.
\end{abstract}

Key Words: Co-author networks; Centrality; h-index.

Received: December 16, 2016 / Revision: January 9, 2017 / Accepted: February 18, 2017

Address for correspondence: Bongseog Kim, Department of Psychiatry, Inje University Sanggye Paik Hospital, 1342 Dongil-ro, Nowon-gu, Seoul 01757, Korea

Tel: +82-2-950-1082, Fax: +82-2-950-8883, E-mail: kimbs328@paik.ac.kr

\section{서 론}

사회 네트워크 분석(social network analysis)이란 관계를 형 성하는 한 쌍 사이에 존재하는 정보인 관계 데이터를 통해 사 회 구성원이 네트워크에서 하는 역할 및 개인이 포함된 네트 워크의 구조 형태를 파악하는 것을 목적으로 한다. ${ }^{1)}$

이러한 사회 네트워크 분석은 각 공저자를 하나의 네트워 크로 표현하는 학술 공동 연구 분야에서도 공저자 네트워크라 는 이름으로 활발히 이루어지고 있으며, 사회 네트워크 분석 을 통해 네트워크 내부의 공저자들의 역할뿐만 아니라 공저

This is an Open Access article distributed under the terms of the Creative Commons Attribution Non-Commercial License (http://creativecommons.org/licenses/by-nc/4.0) which permits unrestricted non-commercial use, distribution, and reproduction in any medium, provided the original work is properly cited.
자들 간의 네트워크 특성 및 구조도 파악할 수 있다. 공저 네 트워크의 노드는 저자(연구자)이며, 링크는 공동 저술, 즉 연 구자들 간의 관계를 나타낸다. 1994년 $\mathrm{Kretschmer}^{2}$ 의 학술지 논문에서 나타나는 공저 네트워크에 대한 연구를 시작으로, 연구자들 간의 사회적 관계 및 학술적 영향력에 대한 분석이 다루어져 왔다. 학술 연구에서 공저자 네트워크 현황을 파악 하는 것은 연구 공동체의 형태 또는 사회적 관계의 경향을 파 악하여, 지식이 생산되고 공유되는 양상을 역동적으로 파악 할 수 있도록 해준다. ${ }^{3)}$

최근에는 모든 학술 분야에서 연구자들 간의 협력 연구가 갈수록 증가하고 있으며, 공저 현상은 2000년대 들어 급격히 증가하는 추세로, ${ }^{4}$ 과학 및 의학 분야에서도 보다 국제적으로 다수의 연구자들의 협력 연구가 증가하고 있다.5) 실제로 정신 
의학 분야에서도 1980 년 3\%에 불과했던 국제 협력 연구가 2011년 들어서는 22\%까지 증가했다고 보고된 바 있으며,) 이 러한 협력 연구의 증가는 학문 분화, 기회시간비용, 연구의 질, 위험 분산 등에 의한 요인들이 작용되는 것으로 알려져 있다. ${ }^{7}$

이러한 국제적 추세로 비추어 보아, 점차 협력 연구의 중요 성은 높아지고 있고 앞으로의 연구 방향 제시를 위해서 현재 국내 학술 연구의 공저 네트워크를 파악하는 것은 큰 의미가 있을 것이다. 의학 분야에서도 공저 네트워크 분석을 통한 협 력 연구 관계를 파악하려는 연구가 이루어지고 있고, 국내 정 신건강의학과에서는 학술지 신경정신의학의 연구 네트워크를 파악하기 위한 시도가 진행된 바 있다. ${ }^{8)}$ 그러나 공동 저술을 통한 연구 형태 파악 및 이를 실제 연구에서 확장시켜 나가기 위해서 보다 다양한 추가 연구를 비롯하여 전문적인 분과 분 야를 다루는 학술지의 분석도 필요할 것으로 생각된다. 이에 본 연구는, 대표적인 국내 분과 전문학회지 중 하나인 학술지 소아청소년정신의학(Journal of the Korean Academy of Child and Adolescent Psychiatry)의 약 10년간의 학술 네트워크를 분석하여, 이를 통해 소아청소년정신건강의학과의 공동 연구 현황에 관한 기초 자료를 제시하고자 한다.

\section{방 법}

\section{대 상}

본 연구는 비교적 좁은 범위로 한정되어 있는 전문학회지의 연구 네트워크 특성을 파악하기 위해서, 대한소아청소년정신 의학회에서 발행하는 학술지 소아청소년정신의학을 분석 대 상으로 하였다. 소아청소년정신의학은 대한민국 정신건강의 학과 학술지 중 대표적인 분과 전문학회지로, 투고논문이 소 아청소년 대상의 정신건강에 관한 연구로 한정되므로 보다 전문적이고 집중된 연구 네트워크에 대해 분석하기에 적합하 다. 또한 2013년부터는 연 4회 학술지가 발행되고, 2014년 국내 정신과학 분야 학술지 17종 중 한국판 과학인용색인(Korean Citation Index, KCI) 영향력 지수가 2번째로 높아) 국내 전문 학술지로서는 비교적 활발한 학술 교류가 이루어져 네트워크 특성이 명확하게 드러날 수 있다.

2005년부터 2015년까지 투고된 논문을 분석 대상으로 선정 하였다. 기간 중 소아청소년정신의학 학술지는 총 34번 발행되 었으며, 251개의 논문이 투고되었다. 논문 투고에 참여한 저 자 수는 564명이었다.

\section{중심성 및 연구 성과}

공저 네트워크를 분석하고자 할 때 파악하는 속성은 일반 적으로 명성, 응집력, 범위, 중개의 4 가지 속성이다. 본 연구에
서는 네트워크 내에서 상대적인 중요성을 측정하는 지표로 가장 많이 사용되는 중심성을 분석하였으며, ${ }^{10)}$ 이 중 연결정도 중심성, 근접 중심성은 명성 속성을, 매개 중심성, 아이겐벡터 중심성은 중개 속성을 나타내는 지표이다.11)

연결정도 중심성(degree centrality)은 네트워크의 저자가 얼 마나 많은 연결을 가지고 있는지 나타내며, 네트워크 중심에 위치하는 정도를 계량화한 것이다. 즉 네트워크 내에서 가지 는 저자의 중요성을 나타낸다. ${ }^{12)}$ 많은 연결을 가지는 저자는 선택의 폭이 넓어 더욱 많은 기회를 가지며, 남에게 덜 의존하 게 되고 더욱 강력한 힘을 가진다고 볼 수 있다. ${ }^{10)}$

근접 중심성(closeness centrality)은 직접적으로 연결된 저 자뿐만 아니라 네트워크 내 간접적으로 연결된 모든 저자 간 의 거리를 측정한 것으로, 한 저자가 얼마나 네트워크의 중앙 에 있는지, 다른 저자들과의 근접 정도를 보여준다. ${ }^{13)}$ 즉 근접 중심성이 높은 저자는 자신이 가진 자원을 가장 빠르게 네트 워크에 확산시킬 수 있고, 다른 저자를 통해 신속하게 정보를 접할 수 있다는 강점이 있다. ${ }^{14)}$

매개 중심성(betweenness centrality)은 네트워크 내에서 한 저자가 중개자 혹은 다리 역할을 얼마나 수행하느냐를 측정 하는 지표로, 중개 역할을 중심으로 간주할 때 사용된다. ${ }^{15}$ 즉, 높은 매개 중심성을 보이는 저자는 네트워크가 전반적으로 잘 연결되어 있다는 것을 의미하는 동시에, 잠재적으로 정보 를 통제 혹은 왜곡할 위험성도 내포한다. ${ }^{16)}$

아이겐벡터 중심성(eigenvector centrality centrality)은 높은 저자 개인의 중심성과 그와 연결관 다른 저자의 중심성 지표 를 함께 고려하는 개념으로, 저자와 연결된 다른 저자의 중심 성이 높으면 해당 저자의 영향력도 더욱 높을 것이라는 의미 이다. 즉, 아이겐벡터 중심성으로 네트워크 내에서 가장 영향 력이 있는 중심 저자를 찾을 수 있다. ${ }^{12}$

저자의 연구 성과는 한국연구재단에 공개되어 있는 연구자 인용정보 중 2004년에서 2014년간의 논문 수, 총 피인용 횟수, 평균 피인용 횟수, $\mathrm{h}$-지수를 이용하였으며, 공개되지 않은 저 자는 제외하였다. ${ }^{9} \mathrm{~h}$-지수는 Hirsch ${ }^{17}$ 가 2005년 개인의 연구 성과 평가에 참조할 수 있는 지표로 제안한 것으로써, 논문 수 와 피인용 수를 모두 고려할 수 있다는 장점이 있다.

\section{네트워크 및 통계 분석}

본 연구는 저자를 노드로, 학술 논문의 공저를 링크로 설 정하여 네트워크를 구성하였다. 네트워크의 분석은 사회 연결 망 분석 전문 프로그램인 Netminer 4.1(Cyram Inc., Seoul, Korea)을 이용하여 네트워크 특성 분석 및 시각화를 하였고, 네트워크 구조가 멱함수 구조를 따르는지 판단하기 위해 적 합도 검정을 시행하였다. ${ }^{18)}$ 
네트워크 분석으로 통해 얻어진 결과를 이용하여 저자가 네트워크 내부에서 가지는 구조적 특성과 실제 연구 성과 간 의 관련성 여부를 확인하기 위해 스피어만 순위 상관분석 (Spearman rank correlation coefficient)을 시행하였다. 통계 분석은 SAS 9.2(SAS Institute Inc., Cary, NC, USA)를 이용 하였고, $95 \%$ 신뢰구간 $(\mathrm{p}<0.05)$ 을 가지도록 하였다.

\section{결 과}

\section{기본 특성}

학술지 소아청소년정신의학의 네트워크 기본 특성은 Table

Table 1. Network characteristics of Journal of the Korean Academy of Child and Adolescent Psychiatry

\begin{tabular}{ll}
\hline \multicolumn{1}{c}{ Characteristic } & Value \\
\hline Number of links & 2597 \\
Density & 0.016 \\
Average degree & 4.605 \\
Mean distance & 3.285 \\
Diameter & 6 \\
\hline
\end{tabular}

1 과 같으며, 분석된 네트워크를 시각화하였다(Fig. 1). 총 8개 의 하위 네트워크가 구성되었으나, 564명의 저자 중 544명이 하나의 네트워크 속에서 연결되었고, 그 외의 7개 네트워크는 1 5명 사이의 소규모 네트워크를 형성하였다. 저자 간의 총 링크 수는 2597이며, 저자 한 명당 가지고 있는 평균 링크 수 는 4.605회로, 이는 한 명의 저자가 평균 4.6명과의 학술 관계 를 맺었음을 의미한다. 저자들 사이의 평균 거리는 3.285 였으 며 네트워크 내부의 저자 사이의 최대 거리는 6이었다.

네트워크를 멱함수의 형태로 분석하였을 때, alpha $=2.559$, $X \min =6$ 으로 나타났으며, $\mathrm{p}<0.01$ 수준에서 멱함수 검정을 시 행한 결과 $p=0.226$ 으로, $p$ 가 0.01 이상이므로 본 네트워크는 유의한 수준에서 멱함수를 따르고 있다고 판단된다. ${ }^{19)}$

\section{네트워크 중심성}

아이겐벡터 중심성을 기준으로 상위 40 명의 중심성과 $\mathrm{h}$-지 수를 Table 2에 기술하였다(단, 한국연구재단에 $\mathrm{h}$-지수가 공 개되어 있지 않은 저자는 표에서 제외함).

저자가 네트워크 내부에서 가지는 구조적 특성과 실제 연

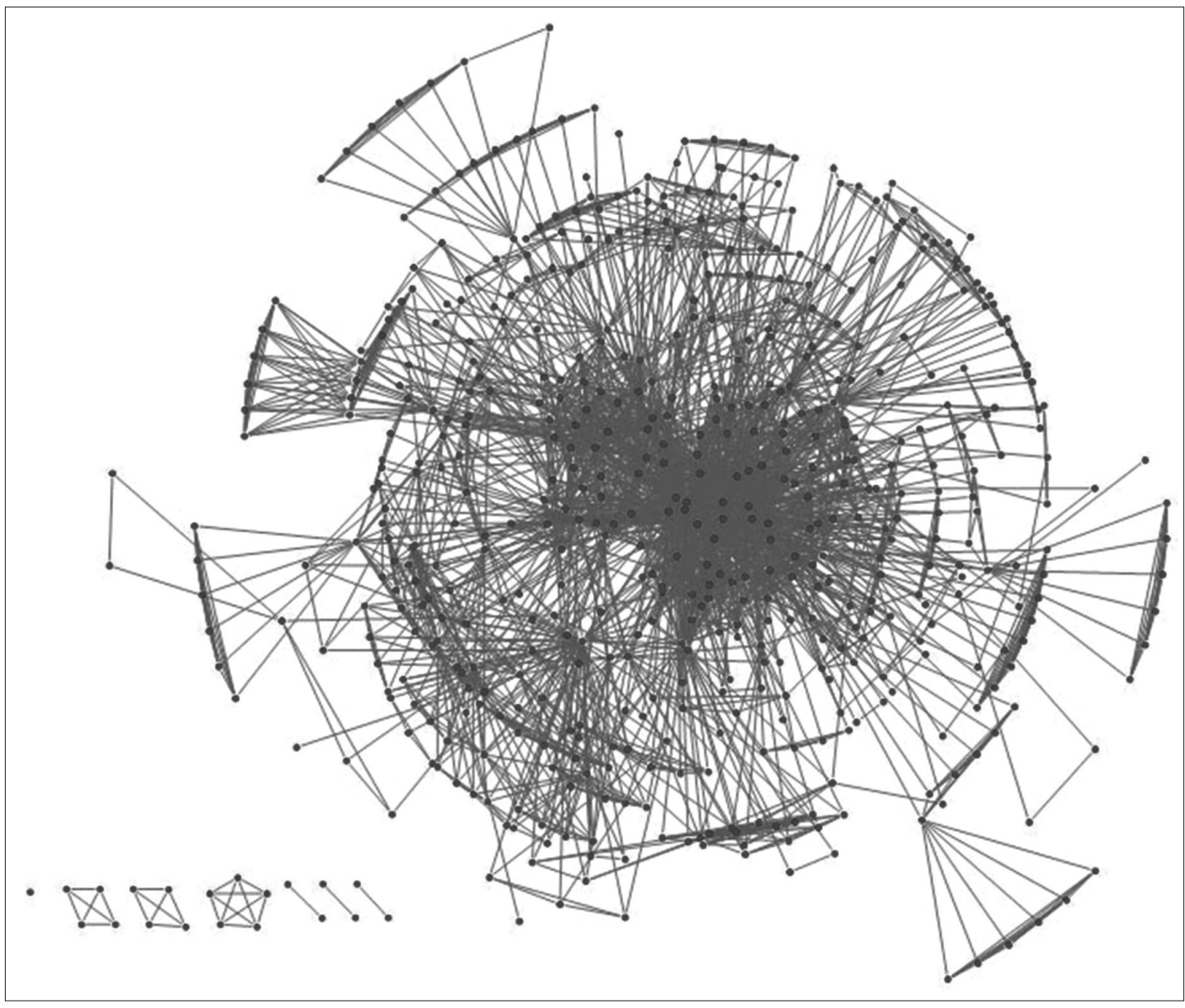

Fig. 1. The structure of co-author networks in "Journal of the Korean Academy of Child and Adolescent Psychiatry" from 2005 to 2015. 
구 성과와의 관련성을 알아보기 위해 스피어만 순위 상관분 석을 시행하였다(Table 3). 대부분의 중심성과 연구 성과 간에 통계적으로 유의한 상관관계가 나타났으나, 평균 피인용 횟수 는 중심성과 통계학적 관련성을 보이지 않았다.

\section{고 찰}

본 연구는 학술지 소아청소년정신의학에 게재된 논문의 공 저 네트워크 현황을 분석하여, 국내 정신건강의학과 소아청소 년 분과에서 이루어지고 있는 연구의 기본 구조 및 저자 간의

Table 2. Centrality scores and research results of the top 40 authors

\begin{tabular}{|c|c|c|c|c|c|c|c|c|c|}
\hline Author & $E C$ & $P C$ & $D C$ & $\mathrm{CC}$ & $B C$ & PCC & CTC & MCC & h-index \\
\hline Author014 & 0.4047 & 8.8121 & 0.1776 & 0.4765 & 0.0875 & 63 & 274 & 4.3 & 7 \\
\hline Author016 & 0.3676 & 7.4253 & 0.1563 & 0.4614 & 0.0567 & 46 & 236 & 5.1 & 6 \\
\hline Author010 & 0.3446 & 7.2520 & 0.1829 & 0.4614 & 0.0915 & 68 & 251 & 3.6 & 8 \\
\hline Author026 & 0.3428 & 7.3964 & 0.1314 & 0.4731 & 0.0986 & 46 & 75 & 1.6 & 4 \\
\hline Author080 & 0.3058 & 6.1829 & 0.1474 & 0.4495 & 0.0530 & 37 & 71 & 1.9 & 5 \\
\hline Author138 & 0.1991 & 3.9294 & 0.0870 & 0.4166 & 0.0371 & 57 & 187 & 3.2 & 6 \\
\hline Author 106 & 0.1835 & 4.2472 & 0.0959 & 0.4220 & 0.0556 & 36 & 57 & 1.5 & 3 \\
\hline Author030 & 0.1828 & 4.6517 & 0.0906 & 0.4461 & 0.0447 & 21 & 73 & 3.4 & 5 \\
\hline Author031 & 0.1756 & 5.0273 & 0.1332 & 0.4735 & 0.0970 & 29 & 70 & 2.4 & 5 \\
\hline Author023 & 0.1755 & 3.1493 & 0.0551 & 0.3817 & 0.0092 & 25 & 62 & 2.4 & 3 \\
\hline Author027 & 0.1411 & 3.0337 & 0.0853 & 0.3905 & 0.0568 & 34 & 40 & 1.1 & 3 \\
\hline Author062 & 0.1380 & 3.6404 & 0.0746 & 0.4196 & 0.0850 & 45 & 117 & 2.6 & 6 \\
\hline Author013 & 0.1211 & 4.6517 & 0.1172 & 0.4353 & 0.0648 & 27 & 71 & 2.6 & 5 \\
\hline Author122 & 0.1194 & 2.8892 & 0.0604 & 0.4143 & 0.0102 & 27 & 98 & 3.6 & 7 \\
\hline Author 166 & 0.1100 & 4.4783 & 0.1012 & 0.4186 & 0.0418 & 48 & 80 & 1.6 & 5 \\
\hline Author197 & 0.1007 & 2.0802 & 0.0693 & 0.3743 & 0.0077 & 32 & 22 & 0.6 & 3 \\
\hline Author169 & 0.0977 & 1.7335 & 0.0462 & 0.3683 & 0.0025 & 14 & 36 & 2.5 & 4 \\
\hline Author232 & 0.0931 & 1.7624 & 0.0515 & 0.3699 & 0.0283 & 16 & 8 & 0.5 & 2 \\
\hline Author 100 & 0.0857 & 2.2825 & 0.0906 & 0.3787 & 0.0629 & 14 & 26 & 1.8 & 3 \\
\hline Author063 & 0.0826 & 2.0225 & 0.0515 & 0.4013 & 0.0203 & 18 & 178 & 9.8 & 3 \\
\hline Author 123 & 0.0822 & 1.9647 & 0.0533 & 0.4041 & 0.0477 & 20 & 67 & 3.3 & 5 \\
\hline Author 175 & 0.0742 & 1.5602 & 0.0533 & 0.3845 & 0.0090 & 4 & 7 & 1.7 & 2 \\
\hline Author054 & 0.0724 & 2.1958 & 0.0639 & 0.3980 & 0.0385 & 48 & 182 & 3.7 & 8 \\
\hline Author060 & 0.0672 & 2.4270 & 0.0693 & 0.3956 & 0.0840 & 32 & 49 & 1.5 & 4 \\
\hline Author049 & 0.0552 & 1.7046 & 0.0568 & 0.3950 & 0.0238 & 32 & 56 & 1.7 & 5 \\
\hline Author137 & 0.0544 & 1.2135 & 0.0249 & 0.3565 & 0.0403 & 12 & 31 & 2.5 & 3 \\
\hline Author139 & 0.0530 & 1.0690 & 0.0160 & 0.3457 & 0.0068 & 11 & 35 & 3.1 & 3 \\
\hline Author290 & 0.0525 & 2.5425 & 0.0782 & 0.3980 & 0.0630 & 18 & 42 & 2.3 & 3 \\
\hline Author058 & 0.0437 & 2.2247 & 0.0817 & 0.4150 & 0.0395 & 35 & 29 & 0.8 & 3 \\
\hline Author389 & 0.0425 & 2.3114 & 0.0586 & 0.3854 & 0.0229 & 13 & 12 & 0.9 & 2 \\
\hline Author034 & 0.0424 & 2.1380 & 0.0817 & 0.4223 & 0.0639 & 20 & 74 & 3.7 & 4 \\
\hline Author178 & 0.0400 & 1.1557 & 0.0586 & 0.3935 & 0.0353 & 21 & 56 & 2.6 & 5 \\
\hline Author402 & 0.0352 & 0.5490 & 0.0231 & 0.3522 & 0.0000 & 6 & 0 & 0 & 0 \\
\hline Author052 & 0.0343 & 0.7223 & 0.0320 & 0.3665 & 0.0016 & 1 & 0 & 0 & 0 \\
\hline Author046 & 0.0314 & 1.5891 & 0.0551 & 0.3840 & 0.0276 & 17 & 20 & 1.1 & 2 \\
\hline Author017 & 0.0308 & 0.5778 & 0.0195 & 0.3558 & 0.0070 & 2 & 36 & 18 & 2 \\
\hline Author212 & 0.0303 & 0.5490 & 0.0266 & 0.3515 & 0.0002 & 2 & 0 & 0 & 1 \\
\hline Author028 & 0.0277 & 2.1380 & 0.0959 & 0.3743 & 0.0905 & 52 & 54 & 1 & 3 \\
\hline Author087 & 0.0271 & 2.0225 & 0.0693 & 0.3859 & 0.0403 & 32 & 161 & 5 & 8 \\
\hline Author392 & 0.0266 & 0.9246 & 0.0515 & 0.3776 & 0.0141 & 38 & 63 & 1.6 & 4 \\
\hline
\end{tabular}

BC: betweenness centrality, CC: closeness centrality, CTC: citation count, DC: degree centrality, EC: eigenvector centrality, MCC: mean citation count, PC: power centrality, PCC: publication count 
Table 3. Spearman rank correlation test for variables

\begin{tabular}{|c|c|c|c|c|c|c|c|c|}
\hline & & 1 & 2 & 3 & 4 & 5 & 6 & 7 \\
\hline 1 & Eigenvector centrality & - & & & & & & \\
\hline 2 & Degree centrality & $0.652^{*}$ & - & & & & & \\
\hline 3 & Closeness centrality & $0.697^{*}$ & $0.837^{*}$ & - & & & & \\
\hline 4 & Betweenness centrality & $0.460^{*}$ & $0.819^{*}$ & $0.729^{*}$ & - & & & \\
\hline 5 & Publication count & $0.512^{*}$ & $0.756^{*}$ & $0.667^{*}$ & $0.617^{*}$ & - & & \\
\hline 6 & Citation count & $0.547^{*}$ & $0.602^{*}$ & $0.765^{*}$ & $0.536^{*}$ & $0.741^{*}$ & - & \\
\hline 7 & Mean citation count & 0.276 & 0.170 & $0.410^{*}$ & 0.217 & 0.200 & $0.740^{*}$ & - \\
\hline 8 & h-index & $0.488^{*}$ & $0.574^{*}$ & $0.691^{*}$ & $0.500^{*}$ & $0.715^{*}$ & $0.882^{*}$ & $0.655^{*}$ \\
\hline
\end{tabular}

*correlation is significant at the 0.01 level $(p<0.01)$

네트워크 특성에 관해 파악하였다.

네트워크 구조를 시각화한 결과에서 알 수 있듯이, 546명의 저자 중 20 명을 제외한 대부분의 저자가 서로 학술적 연결망 을 이루고 있었고 이러한 집중적인 네트워크 구성은 분과 내 부 저자들의 긴밀한 연구 교류가 있음을 보여준다. 저자들 간 의 평균 거리가 3.285 , 최대 거리가 6 이라는 점은 대부분의 저 자들이 3.285번을 거치면 연결될 수 있으며, 최대 6번을 거치면 모든 저자들이 연결될 수 있다는 것을 의미한다. 이전 연구에 따 르면 공동저자들 간 평균 경로거리의 값을 4 9 정도로 제시하 고 있으므로, ${ }^{20)}$ 본 연구의 네트워크는 좁은 세상(small world) 의 특성을 나타낸다고 볼 수 있다. 또한 멱함수 검정을 통해 네 트워크가 멱함수의 특성을 가지는 것도 확인할 수 있었다. 멱 함수의 특성을 가진다는 것은 파레토의 법칙을 따른다고 볼 수 있으므로, ${ }^{21)}$ 상위 $20 \%$ 의 저자가 $80 \%$ 의 저자를 이끌어 가 는, 즉 $20 \%$ 의 저자를 중심으로 연구가 집중되어 있다고 해석 할 수 있다.

공저 네트워크는 사회 네트워크적 측면에서 척도 없는 네트 워크(scale free network) 및 좁은 세상(small world)의 형태를 형성하고 있는 것으로 알려져 있다.22) 이는 소수의 연구자들이 다수의 연구자들의 학술 관계를 만들고 이어주는 허브(hub) 역할을 하고 있다는 것이고, 이는 본 연구에서도 관찰되는 바, 학술지 소아청소년정신의학도 이러한 학술 관계를 따른다고 하겠다.

학술지 소아청소년정신의학의 공저 네트워크의 저자 중심 성 지표들과 저자의 연구 성과는 대부분 통계학적으로 유의 미한 관련성을 보였다. 이는 국내 정신건강의학과의 연구 성 과 수준이 소아청소년정신의학과 분과 내부에서도 유사하게 나타남을 의미함과 동시에, 연구자의 권력 구조 또한 반복되 고 있다고 해석할 수 있다. 그러나 평균 피인용 횟수는 중심성 지표들과 관련성을 보이지 않았는데, 총 논문 수 및 총 인용 횟수는 모든 중심성 지표들과 관련성을 보인 것으로 비추어 보아, 현재 소아청소년정신의학 연구에서의 영향력은 하나의 논문에서 다수의 인용이 발생하는 형태보다, 다수의 논문 게
재를 통한 형태가 주된 것으로 보인다. 즉, 이는 학술지 소아 청소년정신의학의 발전 방향에 대한 참고 지표로서, 평균 피 인용 횟수를 증가시키는 방향으로의 진전이 필요하다고 해석 할 수 있다. 이전 국내에서 시행된 연구인 정신건강의학과 학술 지 '신경정신의학'의 공저 네트워크 분석'에서는 평균 피인용 횟수도 중심성 지표들과 관련성(스피어만 순위 상관분석)을 보였다는 점은, 본 결과가 학술지 소아청소년정신의학에 보다 특이적임을 시사하며, 즉 참고 지표로써의 의미를 둘 수 있겠다.

본 연구의 제한점은 다음과 같다. 먼저, 국내 정신건강의학 과의 전문 분과 학술지를 분석하였다는 점에서 그 의미가 있 으나, 소아청소년정신의학은 정신건강의학과 분과 중에서도 그 전문성이 두드러지는 바, 다른 분과와는 다른 독특한 네트 워크 특성을 가지고 있을 가능성이 있다. 또한, 저자의 연구 성과로 사용한 지표들이 국내 $\mathrm{KCI}$ 등재 논문을 대상으로 계 산된 것으로써, 실제 해외 학술지에 투고가 활발한 현시점에 서 그 저자의 연구 성과를 모두 반영한다고 보긴 힘들다. 같 은 맥락으로 한국연구재단에 자료가 공개되지 않은 저자들은 분석에서 제외되었는데, 지표들이 공개되지 않은 저자가 상대 적으로 연구 활동이 적을 가능성이 크기 때문에 연구 표본에 서 제외되면서 연구 활동이 많은 저자들의 연구 권력을 과소 평가하였을 가능성이 있다. 마지막으로 스피어만 순위 상관 분석은 네트워크 지표 및 저자 연구 성과 간의 관계의 존재여 부만 보여줄 수 있으며, 종속변수에 대한 독립변수의 영향은 알 수 없다. 실제로 이전 선행연구에서 네트워크 지표가 저자 의 연구 성과에 미치는 영향을 파악하기 위해 포아송 분석을 시행한 사례가 있다. ${ }^{8,23}$ 추후 보다 세부적인 통계적 기법을 통 한 분석 및 해석이 연구되어야 할 것으로 보인다.

\section{결 론}

본 연구는 정신건강의학과 분과 학술지인 소아청소년정신 의학에 약 10 년간 게재된 논문을 대상으로 저자들 간의 공저 네트워크를 분석하였고, 공저 관계가 좁은 세상 및 척도 없는 
네트워크를 따르고 있어 타 분야의 공저 현상과 유사함을 밝 혔다. 또한 네트워크 지표 분석을 통해 평균 피인용 횟수의 증

가를 통한 발전적 방향을 제시하였다. 본 연구는 정신건강의 학과의 분과에서 이루어지는 공저 네트워크 분석을 한 기초 연구로서 의미가 있으며, 추후 보다 정교한 기법을 통한 추가 연구가 필요할 것이다.

중심 단어:공저네트워크; 중심성; $\mathrm{h}$-지수.

\section{Conflicts of Interest}

The authors have no financial conflicts of interest.

\section{REFERENCES}

1) Scott J. Social network analysis. 3rd ed. London: SAGE Publications; 2012.

2) Kretschmer H. Coauthorship networks of invisible colleges and institutionalized communities. Scientometrics 1994;30:363-369.

3) Collins HM. The seven sexes: a study in the sociology of a phenomenon, or the replication of experiments in physics. Sociology 1975; 9:205-224.

4) Velden T, Haque A, Lagoze C. A new approach to analyzing patterns of collaboration in co-authorship networks - mesoscopic analysis and interpretation. Scientomterics 2010;85:219-242.

5) Wuchty S, Jones BF, Uzzi B. The increasing dominance of teams in production of knowledge. Science 2007;316:1036-1039.

6) Larivière V, Diepeveen S, Ni Chonaill S, Macaluso B, Pollitt A, Grant J. International comparative performance of mental health research, 1980-2011. Eur Neuropsychopharmacol 2013;23:1340-1347.

7) Choi Y, Lee K. Analysis of types of journal paper coauthor: focused on Korean public administration review (1989-2008). Korean Public Adm Rev 2009;43:51-72.
8) Choi M, Gim M. The characteristic analysis of researches network for journal of Korean Neuropsychiatric Association. J Korean Neuropsychiatr Assoc 2015;54:418-426.

9) Korean Citation Index. National research foundation of Korea [cited 2016 Oct 9]. Available from: https://www.kci.go.kr/.

10) Hanneman RA, Riddle M. Introduction to social network methods. Riverside: University of California, Riverside;2005.

11) Lim BH, Jeon HJ. Using social network analysis to measure network structure of coauthored people in istics logistics: focusing on Korean logistics review. Korea Logist Res Assoc 2011;21:205-229.

12) Bonacich P. Power and centrality: a family of measures. Am J Sociol 1987;92:1170-1182.

13) Okamoto K, Chen W, Li XY. Ranking of closeness centrality for large-scale social networks. In: Preparata FP, Wu X, Yin J, editors. Frontiers in Algorithmics. Berlin: Springer;2008. p.186-195.

14) Marsden PV. Egocentric and sociocentric measures of network centrality. Soc Netw 2002;24:407-422.

15) Son DW. Social network analysis. Seoul: Kyungmoonsa;2002.

16) Freeman LC. Centrality in social networks conceptual clarification. Soc Netw 1978;1:215-239.

17) Hirsch JE. An index to quantify an individual's scientific research output. Proc Natl Acad Sci U S A 2005;102:16569-16572.

18) Cyram N. Cyram netminer 4.1.0. Seoul: Cyram;2013.

19) Clauset A, Shalizi CR, Newman ME. Power-law distributions in empirical data. SIAM Rev 2009;51:661-703.

20) Newman ME. The structure of scientific collaboration networks. Proc Natl Acad Sci U S A 2001;98:404-409.

21) Newman MEJ. Power laws, Pareto distributions and Zipf's law. Contemp Phys 2005;46:323-351.

22) Travers J, Milgram S. An experimental study of the small world problem. Sociometry 1969;32:425-443.

23) Abbasi A, Altmann J, Hossain L. Identifying the effects of co-authorship networks on the performance of scholars: a correlation and regression analysis of performance measures and social network analysis measures. J Inf 2011;5:594-607. 